\title{
INVESTIGATION OF MICROWAVE ENERGY DISTRIBUTION CHARACTER IN A RESONATOR TYPE PLASMATRON
}

\author{
Bordusau S.*, Madveika S., Dostanko A. \\ Belarusian State University of Informatics and Radioelectronics, P.Brovki 6, 220013 Minsk, Belarus \\ * bordusov@bsuir.by
}

\begin{abstract}
An investigation of microwave energy distribution character in a plasma of microwave discharge inside a plasmatron based on a rectangular resonator has been carried out. The experiments were done applying the "active probe" method. Microwave discharge was excited in the air and oxygen. It has been found out that the readings of the "active probe" along the discharge chamber are of periodic character. The readings of the "active probe" and data on the local electric conductivity of plasma obtained using electrical probes have been compared.
\end{abstract}

Keywords: plasma, microwave energy, distribution, resonator, plasmatron.

\section{Introduction}

Because of the necessity to increase the effectiveness of microelectronic devices industrial production it is vital to develop plasma sources that facilitate carrying out processes on larger treated surface areas and with higher technological characteristics. Microwave plasma discharges meet these requirements [1, 2].

The efficiency of plasma formation processes and maintaining of a stable gas discharge greatly depend on the electric component intensity of the electric field $E_{r}$ in the discharge area [3]. Hence, for technological use, microwave resonator type plasmatrons in which a significant increase of electromagnetic wave's field intensity in the plasma formation zone is provided not by the increase of a microwave energy source's power but by design solutions of the microwave field formation system, are of particular interest [4, 5.

Some statements on the development of microwave plasma installations in which rectangular resonators, designed for microwave ovens, are used as microwave applicators, have already been reported 6 6]. Plasma chambers in such discharge systems are characterized by dimensions much bigger than the length of the electromagnetic wave which forms the discharge.

In order to improve the design of discharge systems of this type and to work out recommendations on their application it is necessary to study the peculiarities of plasma formation in them and its electrophysical characteristics.

The question of particular interest is the character of microwave field intensity space distribution in a volume of gas discharge plasma. Earlier we have established that in a microwave discharge system based on a rectangular resonator there is a high electric field intensity in plasma, when the specific microwave power applied to the discharge equals to $W_{\mathrm{MW}}=0.06-0.08 \mathrm{~W} / \mathrm{cm}^{3}[9$. However, up till now there is no any information about the peculiarities of electromagnetic fields in microwave discharge systems of such type.
As it is shown in 10, the character of particles ionization rate distribution in microwave discharge plasma closely correlates with the distribution of field in plasma. The concentration of active plasma particles is connected with the uniformity of treating the materials with plasma. Therefore, the obtained data on the character and particularities of electromagnetic field distribution inside as well as outside of a plasma volume, excited in resonator type plasmatrons, are not only of a scientific but of a practical interest as well.

\section{Experimental apparatus and procedures}

The investigation was performed in a microwave plasmatron based on a rectangular resonator (Fig,1] [11]. The plasma was formed in a cylindrical quartz tube with the external diameter $200 \mathrm{~mm}$ and $340 \mathrm{~mm}$ length placed in the center of a rectangular resonator having the dimensions $34.5 \times 25 \times 38 \mathrm{~cm}$ along the bigger side. An M-112 magnetron, having a matched load output power about $650 \mathrm{~W}$ and generating the oscillations at a frequency $f_{\text {microwave }}=2.45 \pm 0.05 \mathrm{GHz}$, was used as a source of electromagnetic microwave oscillations. The oscillations were introduced into the resonator through a rectangular coupler hole with its long side along the quartz tube (plasma chamber).

To study the microwave energy distribution in a plasma volume of a rectangular resonator we used the so called method of an "active probe", used in microwave devices for measuring the structure of electromagnetic field 12 and the intensity of the electromagnetic radiation [13. This method also has been used in a number of investigations to measure electromagnetic fields in plasma chambers of plasmatrons with various designs of microwave applicators [10, 14, 15].

The method of diagnosing plasma with an "active probe" is based on the registration of radiation and 


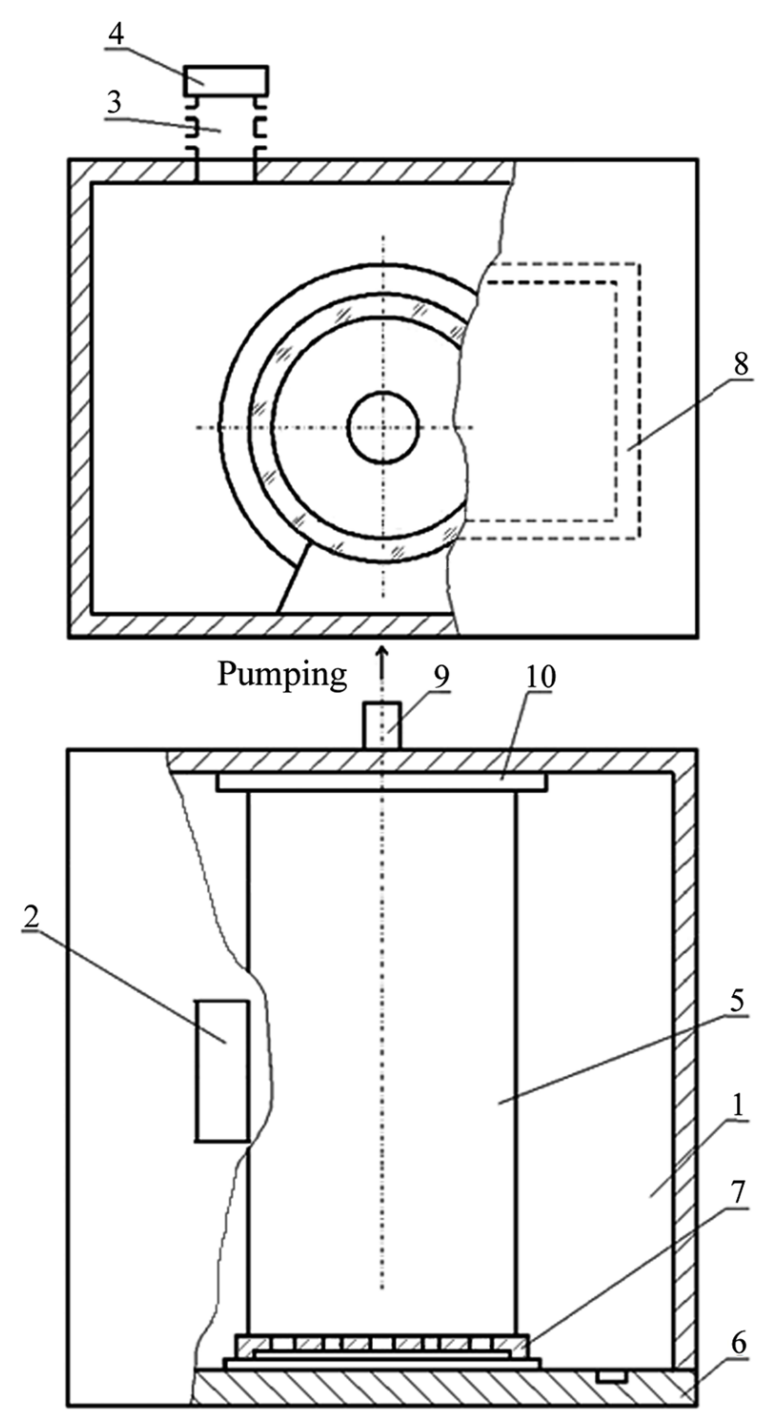

Figure 1. The design of a resonator type plasma discharge block: 1 - rectangular resonator; 2 - coupler hole; 3 - waveguide tract; 4 - magnetron; 5 - quartz plasma chamber; 6 - shutter; 7 - lid of the chamber; 8 - throttle slots; 9 - output pipe; 10 - flange

measuring characteristics of the fields surrounding the probe.

The probe used during microwaves investigations is made in the form of a hard single core coaxial cable with a copper pipe as its external conductor. The cable's wave resistance $\rho=50 \Omega$. From one side of the cable a dipole antenna is formed (Fig. 2). It is a piece of central internal conductor with $1.4 \mathrm{~mm}$ diameter protruding from the external conductor at $l=5 \mathrm{~mm}$, that is much smaller than the length of the electromagnetic wave being investigated. The antenna part of the probe is protected from plasma influence with an additional fluoroplastic insulation in the form of a cap and a quartz cover.

On the other end of the cable, which is outside of the plasma chamber, there is a $50 \Omega$ cable connector. The probe was introduced into the plasma chamber

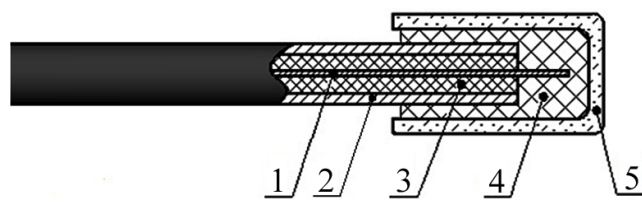

Figure 2. A schematic representation of an antenna's part of the "active probe" used for electric field strength measurements in plasma: 1 - central core; 2 - external conductor; 3 - cable insulation; 4 - additional fluorplastic insulation; 5 - quartz cover

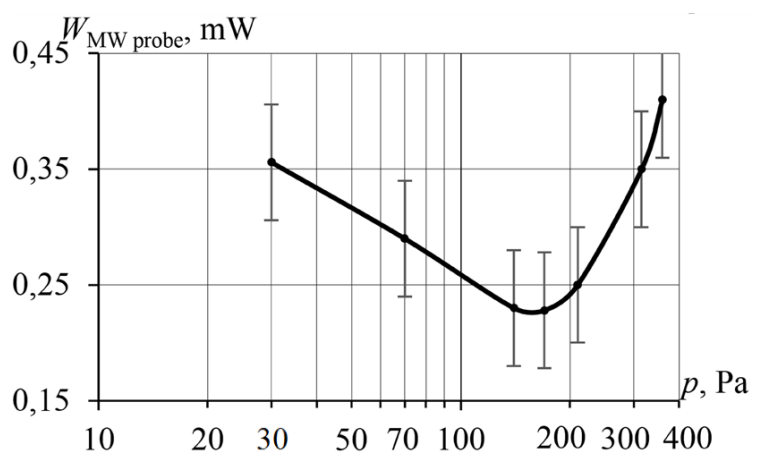

Figure 3. Indications of the "active probe" in $\mathrm{O}_{2}$ discharge depending on the pressure.

through a sealed inlet. During the experiment a microwave signal was induced on the antenna's part of the probe. Its magnitude being proportional to the amplitude of the electric field component $E_{\mathrm{r}}$ directed along the central core of the coaxial cable. In this case, as it is seen from [15], the amount of the microwave signal's power $W_{\text {MWprobe }}$ absorbed by the probe is linearly proportionate to the square of the local magnitude of the electric component of electromagnetic field, i.e.

$$
W_{\mathrm{MW} \text { probe }}=C\left|E_{\mathrm{r}}\right|^{2}
$$

where $C$ is a constant, depending on the probe's design peculiarities.

It gives us the ground to suppose that if such probe is connected with a wattmeter, the wattmeter's readings will characterize the local values of electric field intensity magnitudes. So, moving the probe and measuring the signal $W_{\text {MWprobe }}$ induced on it, it is possible to define the character of microwave energy distribution in space.

To perform the measurements, the probe was connected with the help of a cable connector to a detector section of a M3-51 wattmeter measuring the absorbed power. The principle of operation of this detector is based on the transformation of microwave energy into heat power. The power meter was connected to a PC via an analog-digital converter LA-1,5PCI.

In the process of the investigation, the "active probe" was constantly and continuously moved along the axis of the plasma chamber with the help of an automated system. Its readings were registered along the full length of the plasma chamber. 


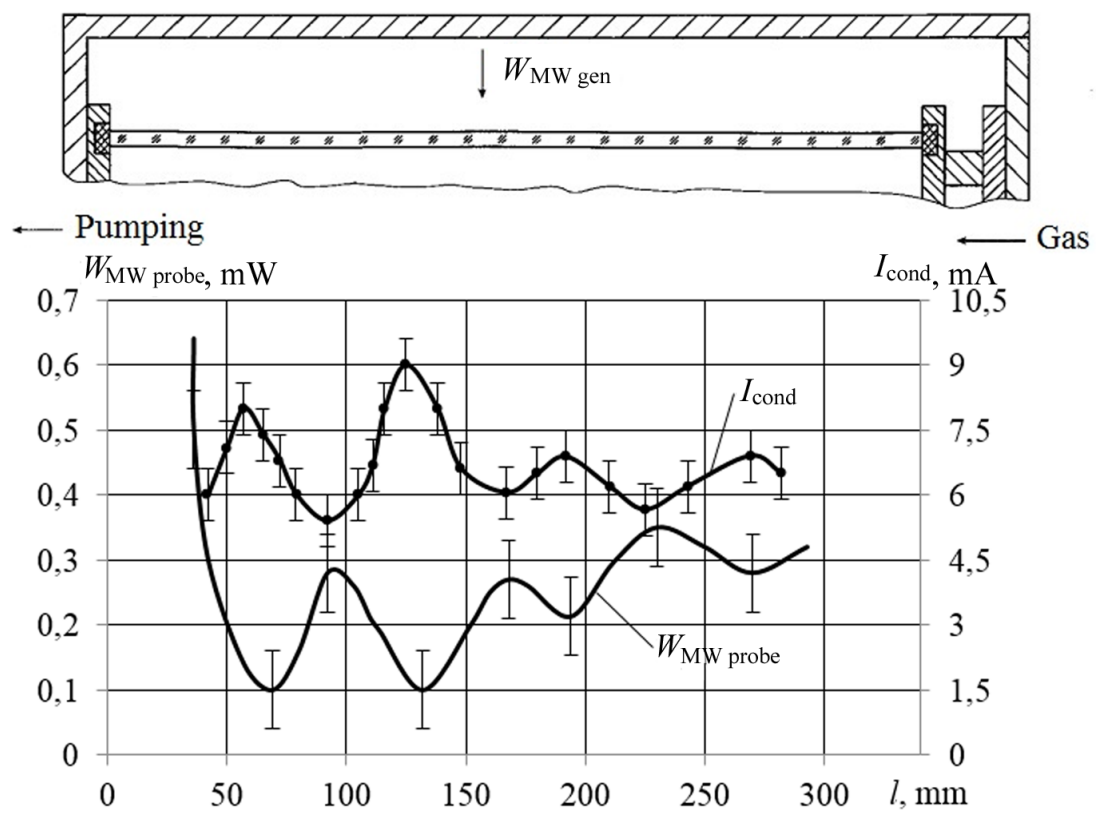

Figure 4. Readings of the active probe $W_{\mathrm{MWprobe}}$ and electric probe Icond in $\mathrm{O}_{2}$ discharge along the plasma chamber l: oxygen pressure $p \approx 140 \mathrm{~Pa}$, microwave generator power $W_{\mathrm{MWgen}}=650 \mathrm{~W} ; W_{\mathrm{MWprobe}}, m W ; I_{\text {cond }}, m A ; l, m m$.

To explain and interpret physically the results obtained with the "active probe" method we also measured the local conductivity of microwave plasma. The measurements were made using a simplified method of electrical probe. The electrical probe was made from two stainless steel wires of $0.5 \mathrm{~mm}$ in diameter protruding from a ceramic insulator by $5 \mathrm{~mm}$. The ceramic insulator was placed in a stainless steel pipe. All the structure was sealed with a compound from the outside. The pipe was also sealed along the outer perimeter in the area of entering the vacuum chamber.

The plasma's local electric conductivity measurements with electrical probe were performed to measure current $I$ appearing in the probe's power supply circuit from a DC source when it contacts with plasma.

The magnitude of plasma electric conductivity is defined by electrons concentration [16]. Because of this, the character of distribution and the magnitude of electric conductivity in the plasma volume give the information about the degree of uniformity of plasma parameters.

Air and oxygen at the pressure of 20-340 Pa were used as plasma forming gases.

\section{Results and discussion}

It has been proved experimentally that the readings of the "active probe" in plasma to a great extent depend on the pressure of plasma forming gas and the location of the probe in the plasma chamber. No significant difference in magnitudes and changes' character of the active probe's readings for plasma discharges in oxygen and air at equal pressure have been observed.

Figure 3 shows the readings of the "active probe" (in power units as per M3-51) within the range of oxygen pressure at constant microwave energy applied to the discharge.

Minimum penetration of microwave field into plasma was registered at the pressure of oxygen $p \approx 140 \mathrm{~Pa}$ which corresponds to the data [3] on optimal breakdown conditions of this gas.

Figure 4 shows the "active probe's" and electrical probe's readings obtained in the oxygen discharge plasma along the plasma chamber's axis. The graphs show the average wattmeter's and milliamperemeter's values obtained in a few experiments.

It can be seen that the changes of the registered values along the plasma chamber are of evident periodic character with the maximum and minimum alternation period of about $70 \mathrm{~mm}$, which is a little bigger than the half length of electromagnetic wave exciting the plasma.

The characteristic type of dependencies also remains for pressure higher and lower than $140 \mathrm{~Pa}$. The difference is in numeric values of the measured magnitudes and increase of difference between minimum and maximum values.

The form of curves' change shown on the figure 4 is also characteristic of air plasma and illustrates a stable form of irregular distribution of microwave energy in the discharge zone volume.

The presented dependencies show that the extreme current values in plasma and the power of the electric field are in the opposite phase, i.e. the discharge zones with local maximum field intensity correspond to minimum electric conductivity and vice versa. This state can be explained by depletion of microwave field depending on the local concentration of electrons (the so called "skin-effect"). In those zones where the electron concentration is higher, the effect of depletion is stronger and vice versa. 
The periodic character of irregularity in the readings of both probes observed during the experiments is presumably connected with the formation of a stable structure of microwave field distribution in the resonator plasmatron chamber volume. This effect should be taken into account while developing technological processes where materials are processed with microwave plasma in large-size plasma chambers of resonator type plasmatrons.

\section{Conclusions}

The performed investigation revealed the occurrence of space irregularity in the microwave energy distribution and local electrical conductivity of plasma in a plasmatron on the base of a resonator chamber in which the resonating volume is partially filled with plasma. In addition to the effect of microwave field penetration into a plasma volume, it should be taken into account when performing the processes of microwave plasma treatment of materials in plasmatrons of this type.

\section{Acknowledgements}

This research has been supported by the State Program of Scientific Research "Physical materials science, new materials and technologies".

\section{References}

[1] Yu.A. Lebedev. Microwave discharges at low pressures and peculiarities of the processes in strongly non-uniform plasma. Plasma Sourses Sci. Technol., 24(5):1-39, 2015.

[2] Bordusov S.V. Microwave plasma technologies in the production of electronic devices. Minsk: Bestprint, 2002.

[3] Mac-Donald A. Microwave Breakdown in Gases. Springer-Verlag, 1969.

[4] Batenin V.M. Klimovski I.I. Lisov G.V. Troitski V.N. Microwave plasma generators: Physics, technology, application. M.: Energoatomizdat, 1988.

[5] Dostanko A. Bordusov S. Svadkovski I. Plasma Processes in Electronics Production, volume 2. Minsk: FUAinform, 2001.

[6] A.I. Kuzmichov. Ion-plasma sources based on microwave ovens. Instruments and experimental techniques, 27(5):648-653, 1995.

[7] Plasma-Preen Plasma Cleaning/Etching System. http://www.plasmapreen.com.

[8] Plasma-Preen Cleaner/Etcher (Patent Pending). http://www.terrauniversal.com

[9] Bordusau S.V. Madveika S.I. Dostanko A.P. Investigation of the microwave electromagnetic field attenuation effect in the discharge chamber of a resonant type plasmatron, 2012. http://ieeexplore.iee.org/ xpl/articleDetails.jsp?arnumber $=6231952$

[10] Nakano T. Samukawa S. The correlation between an electric field and the metastable chlorine ion density distributions in an ultrahigh-frequency plasma. Jap. J. Appl. Phys., 37(5):2686-2692, 1998.
[11] S. Bordusov. Design features of the installation and processes microwave plasma materials processing under low vacuum. Materials, technology, instruments, 6(4):62-64, 2001

[12] Chernushenko A.M. Maiborodin A.V. Parameter measurement electronic devices decimeter and centimeter wave range. M.: Radio and Communication, 1986.

[13] Bilko M.I. Tomaszewski A.K. Measurement of power in the microwave range. M.: Radio and Communication, 1986.

[14] Yasaka Y. Nozaki D. Koga K. et al. Production of large-diameter plasma using multi-slotted planar antenna. Plasma Sources Sci. Technol, 8(4):530-533, 1999.

[15] Mak P. Asmussen J. Experimental investigation of the matching and impressed electric field of a multipolar electron cyclotron resonance discharge. $J$. Vac. Sci. Technol. A., 15(1):154-168, 1997.

[16] Zivotov V.K. Rusanov V.D. Fridman A.A. Diagnostics of nonequilibrium chemically active plasma. M.: Energoatomizdat, 1985. 